\title{
On two-fluid blood flow through stenosed artery with permeable wall
}

\author{
Rupesh K. Srivastav ${ }^{\mathrm{a}, \mathrm{b}, *}$ and V.P. Srivastava ${ }^{\mathrm{a}}$ \\ ${ }^{a}$ Department of Mathematics, Integral University, Lucknow, India \\ ${ }^{\mathrm{b}}$ Department of Mathematics, Ambalika Institute of Management \& Technology, Lucknow, India
}

\begin{abstract}
The present investigation concerns the fluid mechanical study on the effects of the permeability of the wall through an axisymmetric stenosis in an artery assuming that the flowing blood is represented by a two-fluid model. The expressions for the blood flow characteristics, the impedance, the wall shear stress distribution in the stenotic region and the shearing stress at the stenosis throat have been derived. Results for the effects of permeability as well as of the peripheral layer on these blood flow characteristics are quantified through numerical computations and presented graphically and discussed comparatively to validate the applicability of the present model.
\end{abstract}

Keywords: Permeability, darcy number, slip parameter, stenosis throat

\section{Introduction}

The frequently occurring cardiovascular disease, arteriosclerosis or stenosis, responsible for many of the diseases (myocardial infarction, cerebral strokes, angina pectoris, etc.), is the unnatural and abnormal growth that develops at various locations of the cardiovascular system under diseased conditions. Although, the etiology of the initiation of the disease (stenosis) is not well understood but it is well established that once the constriction has developed, it brings about the significant changes in the flow field (pressure distribution, wall shear stress, impedance, etc.). With the knowledge that the hemodynamic factors play an important role in the genesis and the proliferation of arteriosclerosis, since the first investigation of Mann et al. [13], a large number of researcher have addressed the stenotic development problems under various flow situations including Young [27], Young and Tsai [26], Caro et al.

*Corresponding author: Rupesh K. Srivastav, Department of Mathematics, Integral University, Lucknow, India. E-mail: rupeshk. srivastav@gmail.com.
[3], Shukla et al. [21], Liu et al. [11], Srivastava and coworkers [22, 23], Mishra et al. [17], Ponalagusamy [19], Layek et al. [10], Tzirtzilakis [25], Mandal et al. (2007), Politis et al. [18], Medhavi [14-16], Srivastav et al. [22, 24], Naturforschung A. (2013), Eldesoky et al. [5-9], and many others.

The flowing blood has been represented by a Newtonian, non-Newtonian, single or double-layered fluid by the investigators in the literature while discussing the flow through stenoses. It is well known that blood may be represented by a single-layered model in large vessel, however, the flow through the small arteries is known to be a two-layered. Bugliarello and Sevilla [2] and Cokelet [4] have shown experimentally that for blood flowing through small vessels, there is a cell-free plasma (Newtonian viscous fluid) layer and a core region of suspension of all the erythrocytes. Srivastava (2010) concluded that the significance of the peripheral layer increases with decreasing blood vessel diameter. In addition, the endothelial walls are known to be highly permeable with ultra microscopic pores through which filtration occurs. Cholesterol is believed to increase the permeability of the arterial wall. Such 
increase in permeability results from dilated, damaged or inflamed arterial walls. In view of the discussion given above, the research reported here is therefore devoted to discuss the two-layered blood flow through an axisymmetric stenosis in an artery with permeable wall. The mathematical model considers the flowing blood as a two-layered Newtonian fluid, consisting of a core region (central layer) of suspension of all the erythrocytes assumed to be a Newtonian fluid, the viscosity of which may vary depending on the flow conditions and a peripheral region (outer layer) of another Newtonian fluid (plasma) of constant viscosity, in an artery with permeable wall.

\section{Formulation of the problem}

Consider the two-layered axisymmetric flow of blood through an axisymmetric stenosis, specified at the location as shown in Fig. 1. The geometry of the stenosis which is assumed to be manifested in the arterial wall segment is described as

$$
\begin{aligned}
& \frac{\mathrm{R}(\mathrm{z}), \mathrm{R}_{1}(\mathrm{z})}{\mathrm{R}_{0}} \\
& =(1, \beta)-\frac{\left(\delta, \delta_{1}\right)}{2 \mathrm{R}_{0}}\left\{1+\cos \frac{2 \pi}{\mathrm{L}_{0}}\left(\mathrm{z}-\mathrm{d}-\frac{\mathrm{L}_{0}}{2}\right)\right\} \\
& \quad \mathrm{d} \leq \mathrm{z} \leq \mathrm{d}+\mathrm{L}_{0} \\
& =(1, \beta), \quad \text { otherwise }
\end{aligned}
$$

where $\mathrm{z}$ is the axial coordinate, $\left(\mathrm{R}, \mathrm{R}_{1}\right) \cong\left(\mathrm{R}(\mathrm{z}), \mathrm{R}_{1}(\mathrm{z})\right)$ are the radius of the (tube, interface) with constriction; $\mathrm{R}_{0}$ is the radius of the normal (without stenosis) artery; $\mathrm{L}_{0}$ is the stenosis length, $\mathrm{L}$ is the tube length and $\mathrm{d}$ indicates the location of stenosis, $\beta$ is the ratio of

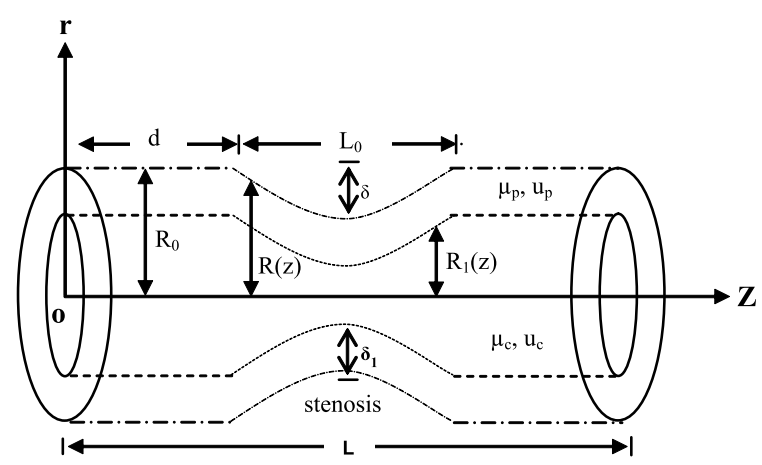

Fig. 1. Two-layered blood flow through an axisymmetric stenosis with permeable wall. the central core radius to the tube radius in the unobstructed region and $\left(\delta, \delta_{1}\right)$ are the maximum height of the stenosis and the bulging of the interface.

The flowing blood is assumed to be represented by a two-layered Newtonian fluid. The equations describing the laminar, steady, one-dimensional flow in the case of a mild stenosis $\left(\delta<<\mathrm{R}_{0}\right)$ are expressed [20] as

$$
\begin{gathered}
\frac{\mathrm{dp}}{\mathrm{dz}}=\frac{\mu_{\mathrm{p}}}{\mathrm{r}} \frac{\partial}{\partial \mathrm{r}}\left(\mathrm{r} \frac{\partial}{\partial \mathrm{r}}\right) \mathrm{u}_{\mathrm{p}}, \quad \mathrm{R}_{1}(\mathrm{z}) \leq \mathrm{r} \leq \mathrm{R}(\mathrm{z}), \\
\frac{\mathrm{dp}}{\mathrm{dz}}=\frac{\mu_{\mathrm{c}}}{\mathrm{r}} \frac{\partial}{\partial \mathrm{r}}\left(\mathrm{r} \frac{\partial}{\partial \mathrm{r}}\right) \mathrm{u}_{\mathrm{c}}, \quad 0 \leq \mathrm{r} \leq \mathrm{R}_{1}(\mathrm{z}),
\end{gathered}
$$

where $R_{1}(z)$ is the radius of the central layer, $\left(u_{p}, \mu_{p}\right)$ and $\left(\mathrm{u}_{\mathrm{c}}, \mu_{\mathrm{c}}\right)$ are (velocity, viscosity) of fluid in the peripheral layer $\left(\mathrm{R}_{1}(\mathrm{z}) \leq \mathrm{r} \leq \mathrm{R}(\mathrm{z})\right)$ and central layer $\left(0 \leq \mathrm{r} \leq \mathrm{R}_{1}(\mathrm{z})\right)$, respectively; $\mathrm{p}$ is the pressure and (r, $\mathrm{z}$ ) are (radial, axial) coordinates in the two-dimensional cylindrical polar coordinate system.

The appropriate boundary conditions [1] for the present problem may be stated [24] as

$$
\begin{gathered}
\frac{\partial \mathrm{u}_{\mathrm{c}}}{\partial \mathrm{r}}=0 \quad \text { at } \mathrm{r}=0 \\
\mathrm{u}_{\mathrm{p}}=\mathrm{u}_{\mathrm{c}} \quad \text { and } \mu_{\mathrm{p}} \frac{\partial \mathrm{u}_{\mathrm{p}}}{\partial \mathrm{r}}=\mu_{\mathrm{c}} \frac{\partial \mathrm{u}_{\mathrm{c}}}{\partial \mathrm{r}} \quad \text { at } \mathrm{r}=\mathrm{R}_{1}(\mathrm{z}),(5) \\
\mathrm{u}_{\mathrm{p}}=\mathrm{u}_{\mathrm{B}} \quad \text { and } \frac{\partial \mathrm{u}_{\mathrm{p}}}{\partial \mathrm{r}}=\frac{\alpha}{\sqrt{\mathrm{k}}}\left(\mathrm{u}_{\mathrm{B}}-\mathrm{u}_{\text {porous }}\right) \quad \text { at } \mathrm{r}=\mathrm{R}(\mathrm{z}),
\end{gathered}
$$

where $\mathrm{u}_{\text {porous }}=-\frac{\mathrm{k}}{\mu_{\mathrm{p}}} \frac{\mathrm{dp}}{\mathrm{dz}}$, is the velocity in the permeable boundary, $\mathrm{u}_{\mathrm{B}}$ is the slip velocity, $\mu_{\mathrm{p}}$ is the plasma viscosity (fluid viscosity in the peripheral layer), $\mathrm{k}$ is Darcy number and $\alpha$ (called the slip parameter) is a dimensionless quantity depending on the material parameters which characterize the structure of the permeable material within the boundary region.

\section{Analysis}

The solutions of Eqs. (2) and (3) under the boundary conditions (4), (5) and (6), yield the expressions for velocity, $\mathrm{u}_{\mathrm{p}}$ and $\mathrm{u}_{\mathrm{c}}$ as 


$$
\begin{array}{r}
\mathrm{u}_{\mathrm{p}}=-\frac{\mathrm{R}_{0}^{2}}{4 \mu_{\mathrm{p}}} \frac{\mathrm{dp}}{\mathrm{dz}}\left\{\left(\frac{\mathrm{R}}{\mathrm{R}_{0}}\right)^{2}-\left(\frac{\mathrm{r}}{\mathrm{R}_{0}}\right)^{2}\right. \\
\left.-2\left(\frac{\mathrm{R}}{\mathrm{R}_{0}}\right)\left(\frac{\sqrt{\mathrm{k}}}{\alpha \mathrm{R}_{0}}\right)+4 \frac{\mathrm{k}}{\mathrm{R}_{0}^{2}}\right\}, \\
\mathrm{u}_{\mathrm{c}}=-\frac{\mathrm{R}_{0}^{2}}{4 \mu_{\mathrm{p}}} \frac{\mathrm{dp}}{\mathrm{dz}}\left\{\left(\frac{\mathrm{R}}{\mathrm{R}_{0}}\right)^{2}-\mu\left(\frac{\mathrm{r}}{\mathrm{R}_{0}}\right)^{2}\right. \\
\left.-(1-\mu)\left(\frac{\mathrm{R}_{1}}{\mathrm{R}_{0}}\right)^{2}\right\} \\
-2\left(\frac{R}{R_{0}}\right)\left(\frac{\sqrt{\mathrm{k}}}{\alpha \mathrm{R}_{0}}\right)+4 \frac{\mathrm{k}}{\mathrm{R}_{0}^{2}},
\end{array}
$$

with $\mu=\mu_{\mathrm{p}} / \mu_{\mathrm{c}}$.

The volumetric flow rate, $\mathrm{Q}$ is now calculated as

$$
\begin{aligned}
& \mathrm{Q}=2 \pi\left\{\int_{0}^{\mathrm{R}_{1}} \mathrm{ru}_{\mathrm{c}} \mathrm{dr}+\int_{\mathrm{R}_{1}}^{R} \mathrm{ru}_{\mathrm{p}} \mathrm{dr}\right\} \\
& \mathrm{Q}=-\frac{\pi \mathrm{R}_{0}^{4}}{8 \mu_{\mathrm{p}}} \frac{\mathrm{dp}}{\mathrm{dz}}\left\{\left(\frac{\mathrm{R}}{\mathrm{R}_{0}}\right)^{4}-(1-\mu)\left(\frac{\mathrm{R}_{1}}{\mathrm{R}_{0}}\right)^{2}\right. \\
&\left.+\frac{8 \mathrm{k}}{\mathrm{R}_{0}^{2}}\left(\frac{\mathrm{R}}{\mathrm{R}_{0}}\right)^{2}-\frac{4 \sqrt{\mathrm{k}}}{\alpha \mathrm{R}_{0}}\left(\frac{\mathrm{R}}{\mathrm{R}_{0}}\right)^{3}\right\},(9)
\end{aligned}
$$

The pressure drop, $\Delta \mathrm{p}(=\mathrm{p}$ at $\mathrm{z}=0,-\mathrm{p}$ at $\mathrm{z}=\mathrm{L})$ across the stenosis in the tube of length, $\mathrm{L}$ is obtained as

$$
\begin{aligned}
\Delta \mathrm{p}= & \int_{0}^{\mathrm{L}}\left(-\frac{\mathrm{dp}}{\mathrm{dz}}\right) \mathrm{dz} \\
= & \frac{8 \mu_{\mathrm{p}} \mathrm{Q}}{\pi \mathrm{R}_{0}^{4}}\left\{\int_{0}^{\mathrm{d}}[\phi(z)]_{\mathrm{R} / \mathrm{R}_{0}=1} \mathrm{dz}+\int_{\mathrm{d}}^{\mathrm{d}+\mathrm{L}_{\mathrm{o}}} \phi(\mathrm{z}) \mathrm{dz}\right. \\
& \left.+\int_{\mathrm{d}+\mathrm{L}_{\mathrm{o}}}^{\mathrm{L}}[\phi(z)]_{\mathrm{R} / \mathrm{R}_{0}=1} \mathrm{dz}\right\}
\end{aligned}
$$

The analytical evaluation of the second integral on the right hand side of Eq. (11) is a formidable task and therefore shall be evaluated numerically, whereas the evaluation of first and third integrals are straight forward. Using now the definitions from the published literature [23, 27], one derives the expressions for the impedance (flow resistance), $\lambda$, the wall shear stress distribution in stenotic region, $\tau_{\mathrm{w}}$ and the shear stress at the stenosis throat, $\tau_{\mathrm{s}}$ in their non-dimensional form as

$$
\lambda=\mu\left\{\frac{\left(1-\mathrm{L}_{0} / \mathrm{L}\right) \eta_{1}}{\eta}+\frac{\eta_{1} \mathrm{~L}_{0}}{2 \pi \mathrm{L}} \int_{0}^{2 \pi} \psi(\theta) \mathrm{d} \theta\right\}
$$

Following the argument $[21,23]$ that the total flux is equal to the sum of the fluxes across the two regions (peripheral and central), one derives the relations: $\mathrm{R}_{1}=\beta \mathrm{R}$ and $\delta_{1}=\beta \delta(0 \leq \beta \leq 1)$. An application of these relations into the Eq. (9), yields

$$
\frac{\mathrm{dp}}{\mathrm{dz}}=-\frac{8 \mu_{\mathrm{p}} \mathrm{Q}}{\pi \mathrm{R}_{0}^{4}} \phi(z),
$$

where

$$
\begin{aligned}
\phi(z)=1 /\left\{\left[1-(1-\mu) \beta^{4}\right]\left(\mathrm{R} / \mathrm{R}_{0}\right)^{4}\right. \\
\left.+8 \mathrm{k}\left(\mathrm{R} / \mathrm{R}_{0}\right)^{2} / \mathrm{R}_{0}^{2}-4 \sqrt{\mathrm{k}}\left(\mathrm{R} / \mathrm{R}_{0}\right)^{3} / \alpha \mathrm{R}_{0}\right\} .
\end{aligned}
$$

where

$$
\tau_{\mathrm{s}}=\left[\tau_{\mathrm{w}}\right]_{R / R_{0}=1-\delta / R_{0}}
$$

$$
\begin{gathered}
\psi(\theta)=[\phi(\mathrm{z})]_{R / R_{0}=a+b \cos \theta}, \mathrm{a}=1-\delta / 2 \mathrm{R}_{0}, \\
\mathrm{~b}=\delta / 2 \mathrm{R}_{0} \\
\eta_{1}=1+8 \mathrm{k} / \mathrm{R}_{0}^{2}-4 \sqrt{\mathrm{k}} / \alpha \mathrm{R}_{0}, \\
\eta=1-(1-\mu) \beta^{4}+8 \mathrm{k} / \mathrm{R}_{0}^{2}-4 \sqrt{\mathrm{k}} / \alpha \mathrm{R}_{0}, \\
\lambda=\bar{\lambda} / \lambda_{0},\left(\tau_{\mathrm{w}}, \tau_{\mathrm{s}}\right)=\left(\bar{\tau}_{\mathrm{w}}, \bar{\tau}_{\mathrm{s}}\right) / \tau_{0}
\end{gathered}
$$

$\lambda_{0}=8 \mu_{\mathrm{c}} \mathrm{L} / \eta_{1} \pi \mathrm{R}_{0}^{4}$ and $\tau_{0}=4 \mu_{\mathrm{c}} \mathrm{Q} / \eta_{1} \pi \mathrm{R}_{0}^{3}$ are the flow resistance and shear stress, respectively for a single-layered Newtonian fluid in a normal artery (no stenosis) with permeable wall and $\left(\bar{\lambda}, \bar{\tau}_{\mathrm{w}}, \bar{\tau}_{\mathrm{s}}\right)$ are 
respectively, (the impedance, the wall shear stress and the shearing stress at stenosis throat) obtained from the definitions [27]:

$$
\begin{array}{r}
\bar{\lambda}=\Delta \mathrm{p} / \mathrm{Q}, \quad \bar{\tau}_{\mathrm{w}}=-(\mathrm{R} / 2) \mathrm{dp} / \mathrm{dz} \\
\text { and } \quad \bar{\tau}_{\mathrm{s}}=\left[\bar{\tau}_{\mathrm{w}}\right]_{\mathrm{R} / \mathrm{R}_{0}=1-\delta / \mathrm{R}_{0} .}
\end{array}
$$

\section{Numerical results and discussion}

To discuss the results of the study quantitatively, computer codes are developed to evaluate the analytical result for flow resistance, $\lambda$, the wall shear stress, $\tau_{\mathrm{w}}$, and shear stress at the stenosis throat, $\tau_{\mathrm{s}}$ obtained above in Equations (12)-(14) for various parameter values and some of the critical results are displayed graphically in Figs. 2-13. The various parameters are selected $[1,24,27]$ as: $\mathrm{L}_{0}(\mathrm{~cm})=1$; $\mathrm{L}(\mathrm{cm})=1,2,5,10 ; \alpha=0.1,0.2,0.3,0.5 ; \sqrt{\mathrm{k}}=$ $0,0.1,0.2,0.3,0.4,0.5 ; \beta=1,0.95,0.90 ; \mu=$ $1,0.5,0.3,0.1 ;$ and $\delta / \mathrm{R}_{0}=0,0.5,0.10,0.45$, 0.20 ; etc. It is worth mentioning here that present study corresponds to impermeable artery case, to singlelayered model study, and to no stenosis case for parameter values $\sqrt{\mathrm{k}}$ (here and after called Darcy number) $=0 ; \beta=1$ or $\mu=1$, and $\delta / \mathrm{R}_{0}=0$; respectively.

The flow resistance $\lambda$, increases with the stenosis height, $\delta / \mathbf{R}_{0}$, for any given set of parameters. At any given stenosis height, $\delta / \mathrm{R}_{0}, \lambda$ decreases with the peripheral layer viscosity, $\mu$ from its maximal magnitude obtained in a single-layered study (i.e., $\mu=1$ or $\beta=1$; Fig. 2).

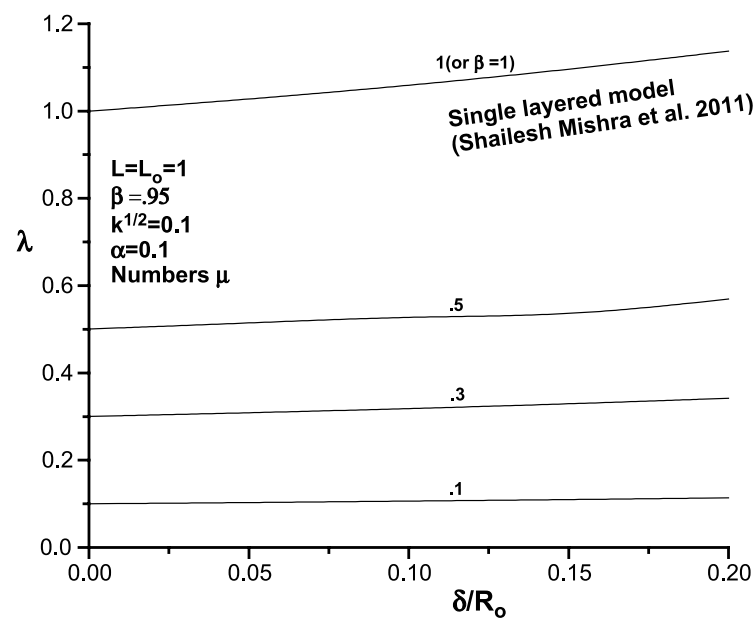

Fig. 2. Impedance, $\lambda$ vs. stenosis height $\delta / \mathbf{R}_{\mathrm{o}}$ for different $\mu$.

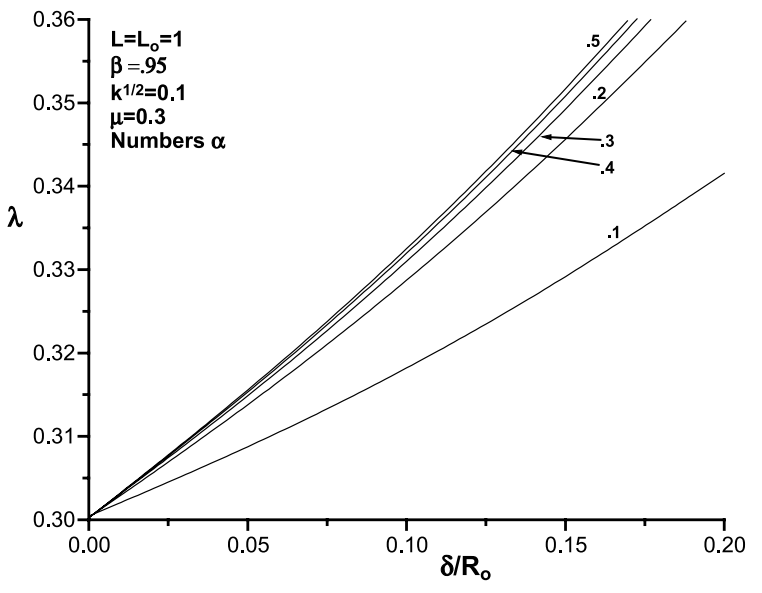

Fig. 3. Impedance, $\lambda$ vs. stenosis height $\delta / \mathrm{R}_{\mathrm{o}}$ for different $\alpha$.

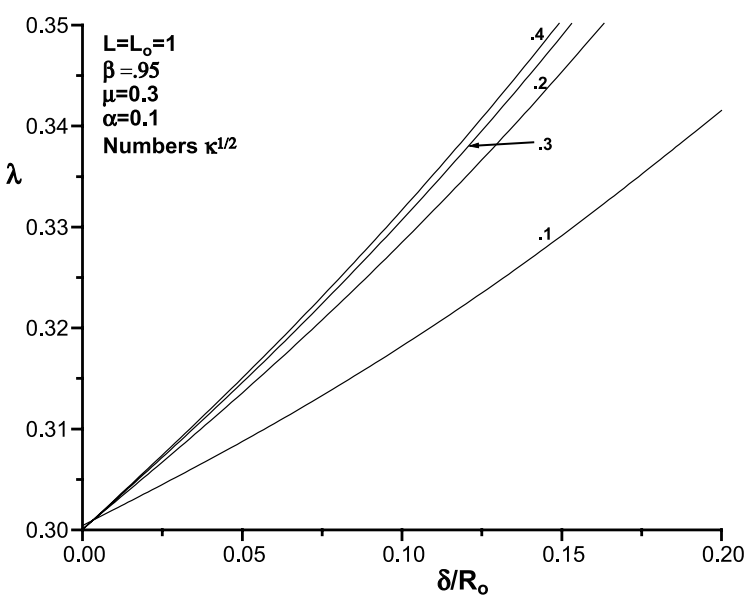

Fig. 4. Impedance, $\lambda$ vs. stenosis height $\delta / \mathrm{R}_{\mathrm{o}}$ for different $\kappa^{1 / 2}$.

One observes that at any given stenosis height, $\delta / \mathrm{R}_{0}$, the impedance, $\lambda$ increases with the slip parameter, $\alpha$ (Fig. 3). The blood flow characteristic, $\lambda$ increases with the Darcy number, $\sqrt{\mathrm{k}}$ at any given stenosis height, $\delta / \mathrm{R}_{0}$ (Fig. 4). The impedance, $\lambda$ decreases with increasing tube length $\mathrm{L}$ which inturns implies that $\lambda$, increases with increasing value of $\mathrm{L}_{0} / \mathrm{L}$ (stenosis length, Fig. 5).

One observes that the flow resistance, $\lambda$ decreases rapidly with increasing value of the Darcy number, $\sqrt{\mathrm{k}}$ from its maximal magnitude at $\sqrt{\mathrm{k}}=0$ (impermeable wall) in the range $0 \leq \sqrt{\mathrm{k}} \leq 0.15$ and afterwards assumes an asymptotic value with increasing values of the Darcy number, $\sqrt{\mathrm{k}}$ (Fig. 6). We notice that the 


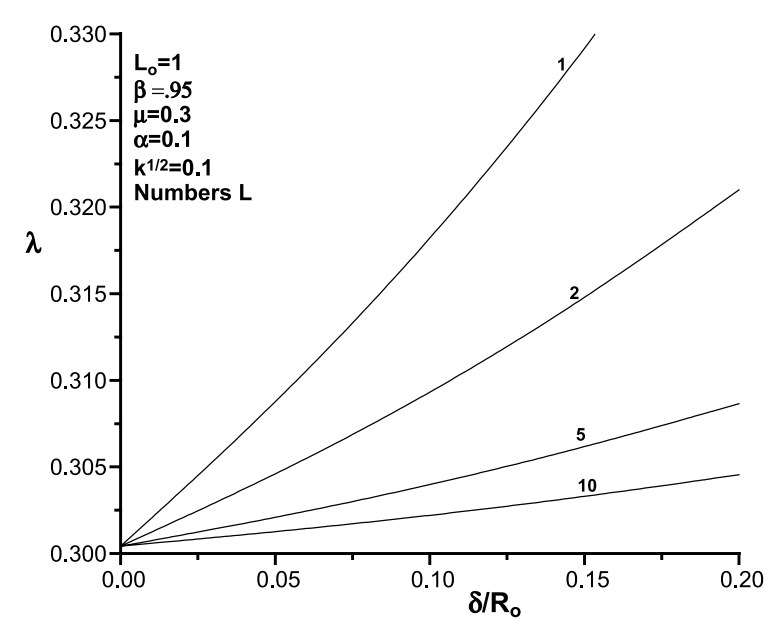

Fig. 5. Impedance, $\lambda$ vs. stenosis height $\delta / \mathrm{R}_{\mathrm{o}}$ for different $\mathrm{L}$.

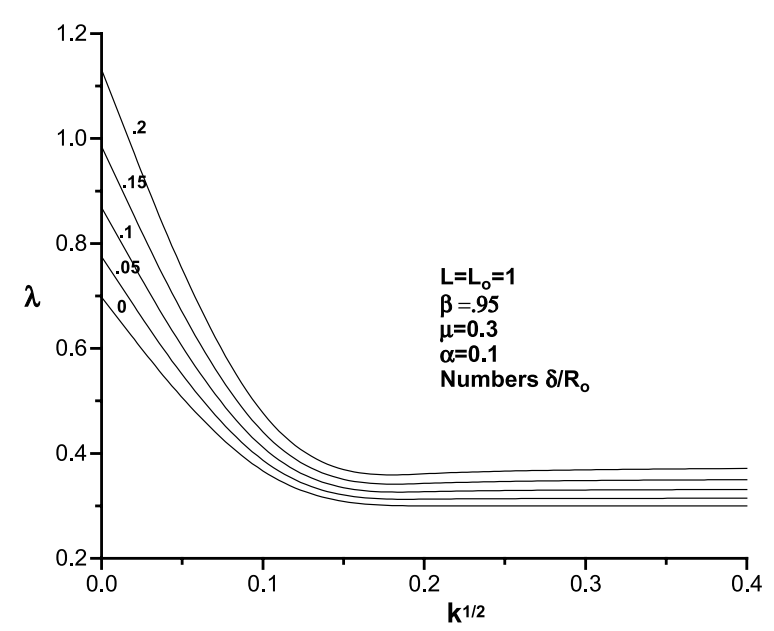

Fig. 6. Impedance, $\lambda$ versus Darcy number $\kappa^{1 / 2}$ for different $\delta / R_{0}$.

blood flow characteristic, $\lambda$ increases with the slip parameter, $\alpha$ from its minimal magnitude at $\alpha=0.1$ and approaches to an asymptotic magnitude when $\alpha$ increases from 0.2 (Fig. 7).

The wall shear in the stenotic region, $\tau_{\mathrm{w}}$ increases from its approached value at $\mathrm{z} / \mathrm{L}_{0}=0$ to its peak value at $\mathrm{z} / \mathrm{L}_{0}=0.5$ and then decreases from its peak value to its approached value at the end point of the constriction profile at $\mathrm{z} / \mathrm{L}_{0}=1$ for any given set of parameters (Figs. 8-11). The blood flow characteristic, $\tau_{\mathrm{w}}$ decreases with the peripheral layer viscosity, $\mu$ at any axial location of the constriction profile (Fig. 8). At any point of stenotic region, the wall shear stress, $\tau_{\mathrm{w}}$ increases with Darcy number, $\sqrt{\mathrm{k}}$ (Fig. 9). The flow characteristic $\tau_{\mathrm{w}}$ also increases with the slip param-

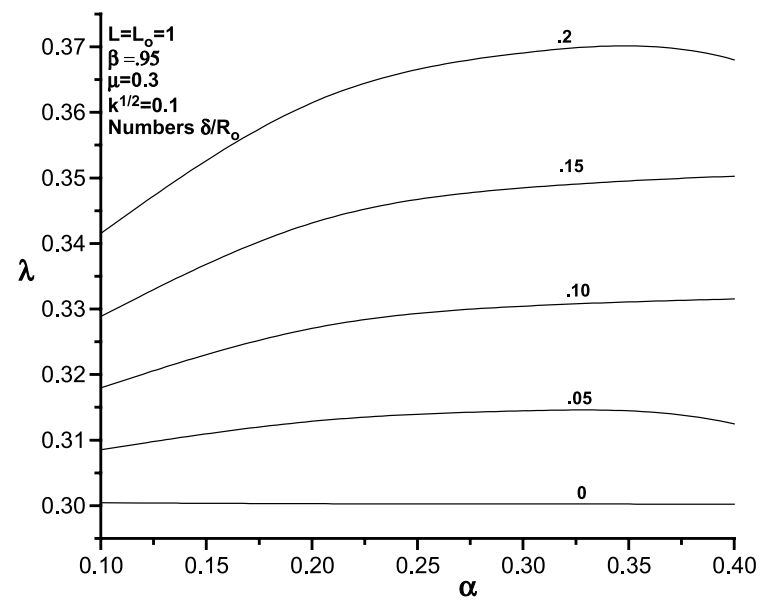

Fig. 7. Impedance, $\lambda$ versus slip parameter, $\alpha$ for different $\delta / R_{0}$.

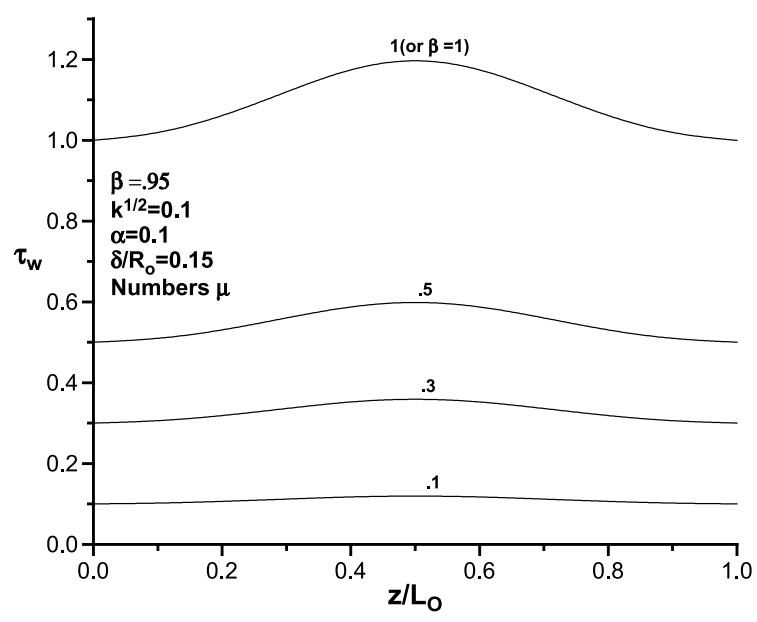

Fig. 8. Wall shear stress in the stenotic region for different $\mu$.

eter, $\alpha$ at any axial location in the stenotic region (Fig. 10).

For any given set of parameters, the wall shear stress, $\tau_{\mathrm{w}}$ increases with the stenosis height, $\delta / \mathrm{R}_{0}$ (Fig. 12). The blood flow characteristic, $\tau_{\mathrm{s}}$ increases with the slip parameter, $\alpha$ (Fig. 13) for any given set of other parameters. Numerical results reveal that the variations of the shear stress, $\tau_{\mathrm{s}}$ are similar to that of the impedance (flow resistances), $\lambda$ with respect to any parameter.

\section{Conclusions}

To observe the effects of the permeability of the artery wall and the peripheral layer on blood flow 


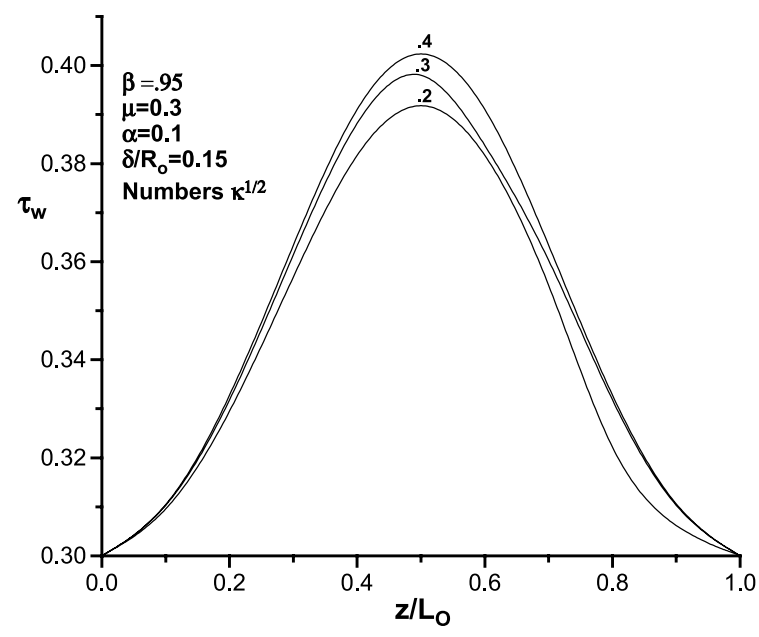

Fig. 9. Wall shear stress in the stenotic region for different $\kappa^{1 / 2}$.

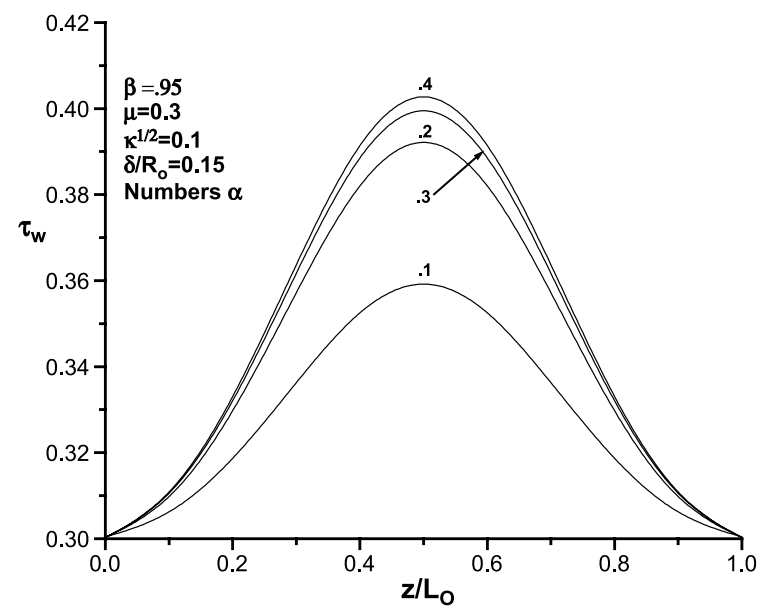

Fig. 10. Wall shear stress in the stenotic region for different $\alpha$.

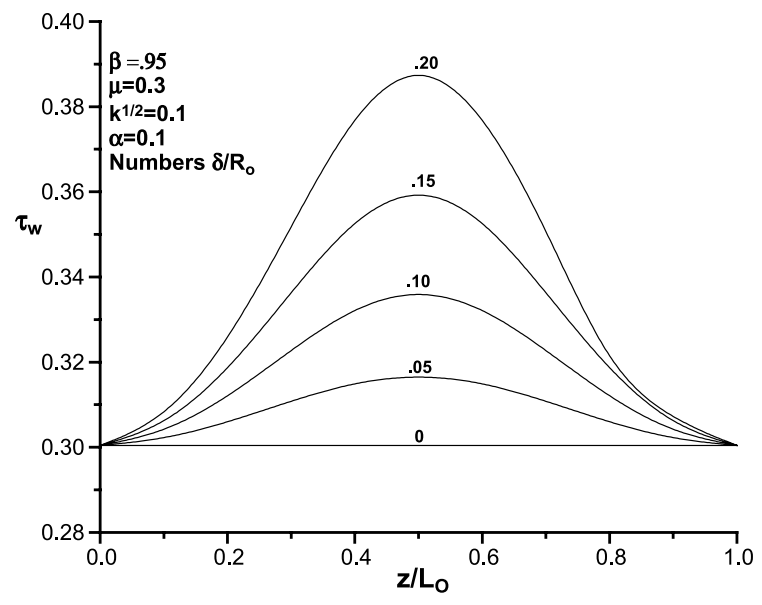

Fig. 11. Wall shear stress in the stenotic region for different $\delta / R_{0}$.

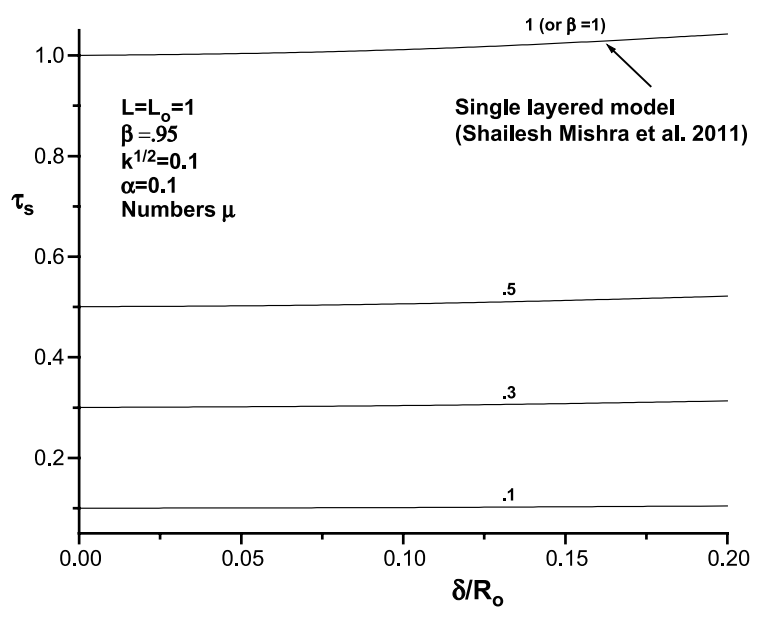

Fig. 12. Shear stress at stenosis throat, $\tau_{\mathrm{s}}$ versus stenosis height, $\delta / \mathrm{R}_{\mathrm{o}}$ for different $\mu$.

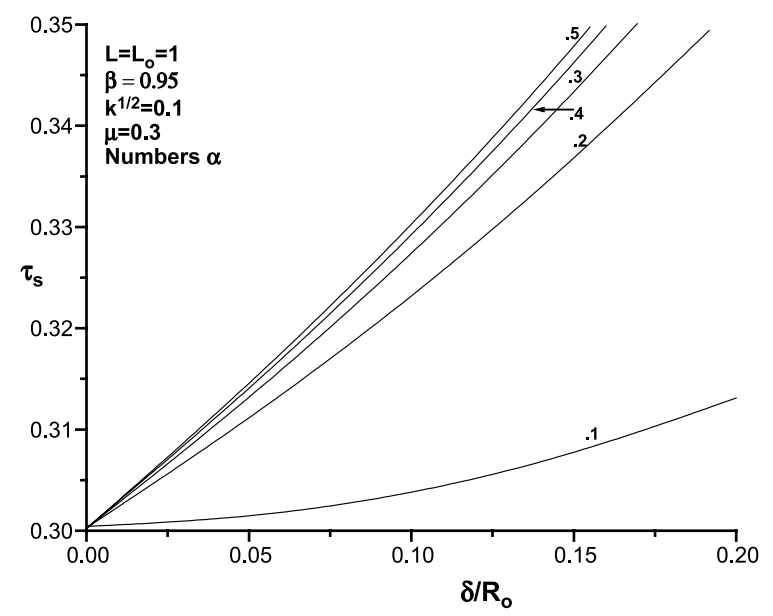

Fig. 13. Shear stress at stenosis throat, $\tau_{\mathrm{s}}$ versus stenosis height, $\delta / \mathrm{R}_{\mathrm{o}}$ for different $\alpha$.

characteristics due to the presence of a stenosis, a two-fluid blood flow of Newtonian fluid through an axisymmetric stenosis in an artery with permeable wall has been studied. The study enables one to observe the simultaneous effects of the wall permeability and the peripheral layer on blood flow characteristics due to the presence of a stenosis. For any given set of parameters, the blood flow characteristics (impedance, wall shear stress, etc.) assume lower magnitude in two-fluid model than its corresponding value in one-fluid analysis. The impedance decreases with increasing Darcy number from its maximal magnitude in the case of impermeable wall (i.e., at zero Darcy number). It is 
therefore concluded that the existence of permeability in the artery wall and the presence of the peripheral layer in the artery helps the functioning of the diseased artery.

\section{References}

[1] G.S. Beavers and D.D. Joseph, Boundary conditions at a naturally permeable wall, J Fluid Mech 30(1) (1967), 197-207.

[2] G. Bugliarello and Sevilla, Velocity distribution and other characteristics of steady and pulsatile blood flow in fine glass tubes, Biorheol 7 (1970), 85-107.

[3] C.G. Caro, T.J. Pedley, R.C. Schroter and W.A. Seed, The Mechanics of the Circulation, Oxford Medical, N.Y., 1978.

[4] G.R. Cokelet, The Rheology of Human Blood, In: Biomechanics, Prentice-Hall, Englewood Cliffs, N.J., 1972.

[5] I.M. Eldesoky, Slip effects on the unsteady MHD Pulsatile blood flow through porous medium in an artery under the effect of body acceleration, International Journal of Mathematics and Mathematical sciences (in press), 2012a.

[6] I.M. Eldesoky, Influence of slip condition on peristaltic transport of a compressible Maxwell fluid through porous medium in a tube, International Journal of Applied Mathematics and Mechanics 8(2) (2012b), 177.

[7] E.F. EI-Shehawey, N.T. Dabe and I.M. Eldesoky, Slip effects on peristaltic flow of a non-Newtonian Maxwellian fluid, Journal of Acta Mechanica 186 (2006), 141-159.

[8] I.M. Eldesoky, M.H. Kernel and R.M. Hussien, Abumandour, Numerical study of unsteady MHD pulsatile flow through porous medium in an artery using Generalized Differential Quadrature Method, International Journal of Materials, Mechanics and Manufacturing 1(2), 2013.

[9] I.M. Eldesoky, Unsteady MHD pulsatile blood flow through porous medium in a stenotic channel with slip at the permeable walls subjected to time dependent velocity (injection/suction), Walailak Journal of Science and Technology 11(12), 2014.

[10] G.C. Layek, S. Mukhopadhyay and R.S.R. Gorla, Unsteady viscous flow with variable viscosity in a vascular tube with an overlapping constriction, Int J Engg Sci 47 (2009), 649-659.

[11] G.T. Liu, X.J. Wang, B.Q. Ai and L.G. Liu, Numerical study of pulsating flow through a tapered artery with stenosis, Chin Journal Phys 42 (2004), 401-409.

[12] P.K. Mandal, S. Chakravarty and Mandal, A Numerical study on the unsteady flow of non-Newtonian fluid through diffrerently shaped arterial stenosis, Int J Comput Math 84 (2007), 1059-1077.

[13] F.C. Mann, J.F. Herrick, H.E. Essex and E.J. Blades, Effects on blood flow of decreasing the lumen of blood vessels, Surgery 4 (1938), 249-252.
[14] A. Medhavi, R.K. Srivastav, Q.S. Ahmad and V.P. Srivastava, Two-phase arterial blood flow through a composite stenosis, e-Journal of Science and Technology 7(4) (2012), 83-94.

[15] A. Medhavi, A macroscopic two-phase blood flow through a stenosed artery with permeable wall, Appl Bionics and Biomechanics 10(1) (2013), 11-18.

[16] A. Medhavi, On macroscopic two-phase arterial blood flow through an overlapping stenosis, e-Journal of Science and Technology 6 (2011), 19-31.

[17] J.C. Misra and G.C. Shit, Blood flow through arteries in a pathological state: A theoretical study, Int J Engg Sci 44 (2006), 662-671.

[18] A.K. Politis, G.P. Stavropoulos, M.N. Christolis, F.G. Panagopoulos, N.S. Vlachos and N.C. Markatos, Numerical modeling of simulated blood flow in idealized composite arterial coronary grafts: Transient flow, J Biomechanics 41(1) (2008), 25-39.

[19] R. Ponalagusamy, Blood flow through an artery with mild stenosis: A two layered model, different shapes of stenosis and slip velocity at the wall, J Appl Sci 7(7) (2007), 1071-1077.

[20] M. Sharan and A.S. Popel, A two-phase model for flow of blood in narrow tubes with increased viscosity near the wall, Birheol 38 (2001), 415-428.

[21] J.B. Shukla, R.S. Parihar, S.P. Gupta, Effects of peripheral layer viscosity on blood flow thorough the arterywith mild stenosis, Bull Math Biol 42 (1980), 797-805.

[22] R.K. Srivastav, Q.S. Ahmad and A.W. Khan, Two-phase model of blood flow through a composite stenosis in the presence of a peripheral layer, Journal of Multidisciplinary Scientific Research, (Africa) 1(5) (2013), 39-45.

[23] V.P. Srivastava, R. Rastogi and R. Vishnoi, A two-layered suspension blood flow through an overlapping stenosis, Computers and Mathematics with Applications 60 (2010), 432-441.

[24] V.P. Srivastava, M. Tandon and R.K. Srivastav, A macroscopic two-phase blood flow through a bell shaped stenosis in an artery with permeable wall, Applications and Applied Mathematics 7(1), (2012), 37-51.

[25] E.E. Tzirtzilakis, Biomagnetic fluid flow in a channel with stenosis, Physica D 237 (2008), 66-81.

[26] D.F. Young and F.Y. Tsai, Flow characteristics in model of arterial stenosis - steady flow, Journal of Biomechanics, 6 (1973), 395-410.

[27] D.F. Young, Effects of a time-dependent stenosis of flow through a tube, Journal of Eng Ind 90 (1968), 248-254.

[28] A. Zeitschrift Fur Naturforschung, Theoretical study of unsteady blood flow of Jeffery fluid through stenosed arteries with permeable walls 68a (2013), 489-498. 

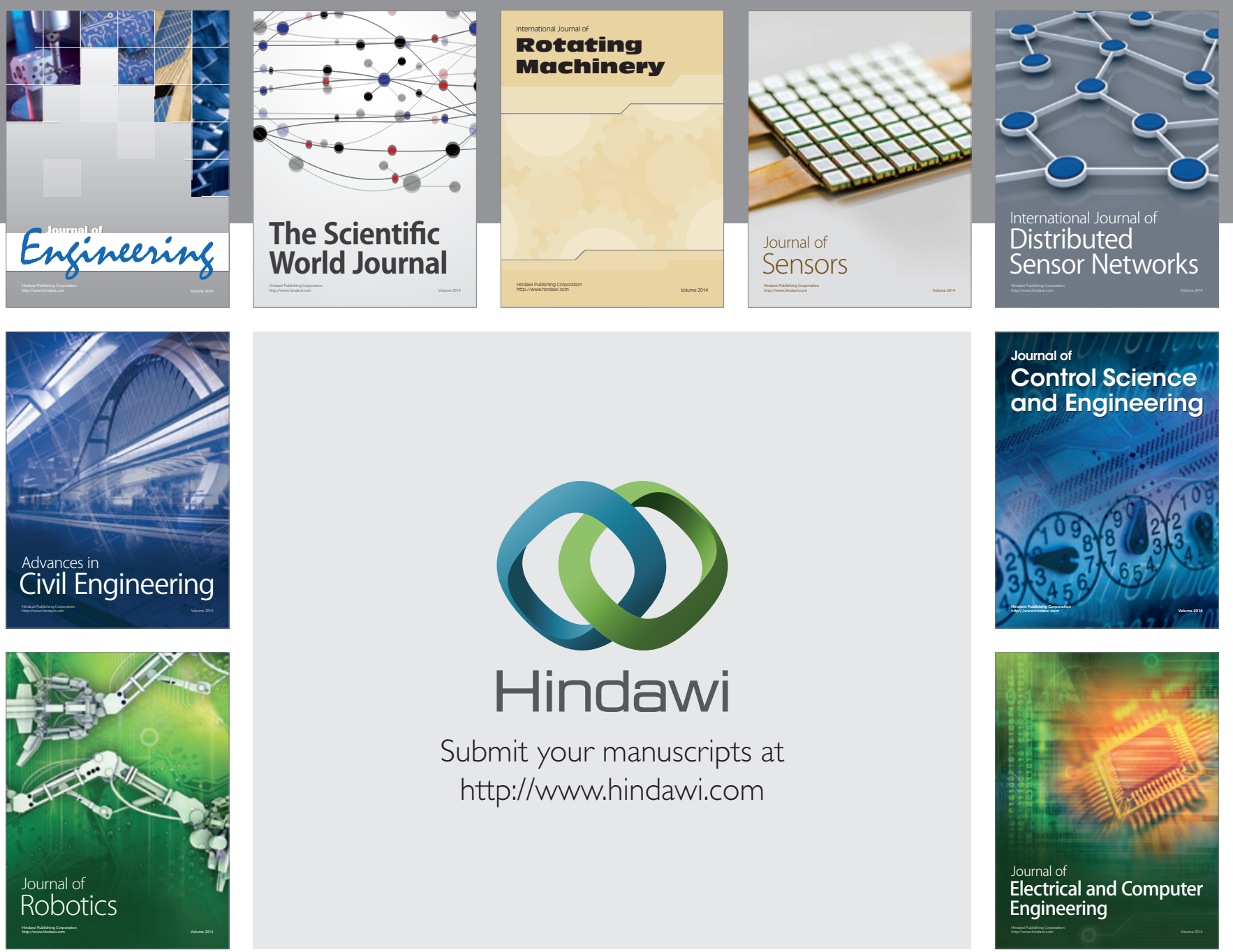

Submit your manuscripts at

http://www.hindawi.com
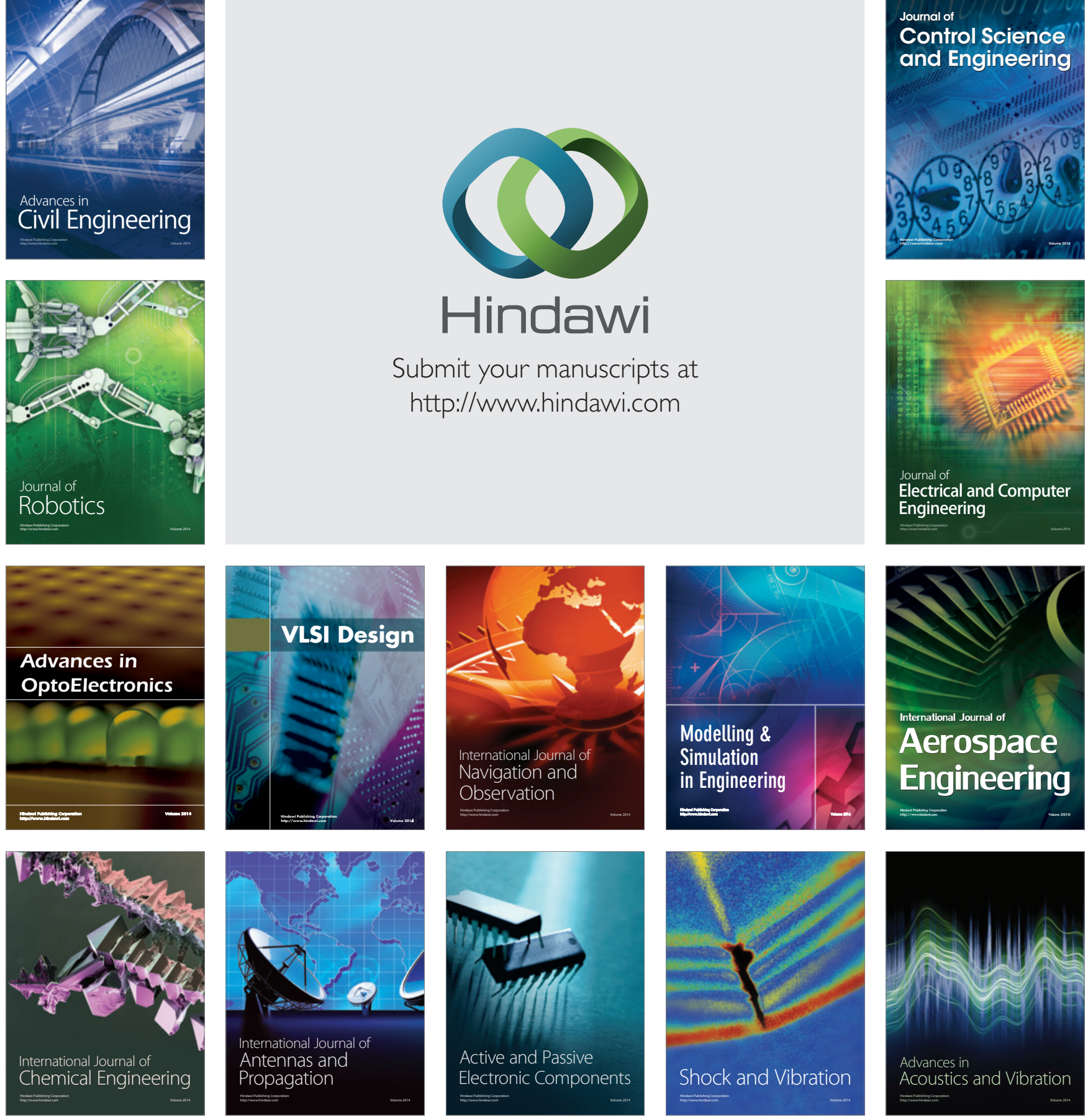